\title{
Embolization of a third ventricular choroid plexus papilloma: illustrative case
}

\author{
Jordan C. Xu, MD, ${ }^{1}$ Shuichi Suzuki, MD, PhD, ${ }^{1,2}$ Jennifer Chrislip, PA-C, ${ }^{2}$ Ali Nael, MD, ${ }^{3}$ and William G. Loudon, MD, PhD ${ }^{2}$ \\ ${ }^{1}$ Department of Neurosurgery, University of California, Irvine Medical Center, Orange, California; ${ }^{2}$ Department of Neurosurgery, Children's Hospital of Orange County, Orange, \\ California; and ${ }^{3}$ Department of Pathology, University of California Irvine and Children's Hospital of Orange County, Orange, California
}

\begin{abstract}
BACKGROUND Choroid plexus tumors are rare neoplasms that are typically found in the lateral ventricles. They have infrequently been reported in the third ventricle, and treatment strategies have varied.

OBSERVATIONS The authors described a 6-month-old patient who presented with irritability and hypotonia. The patient was found to have a large tumor in the third ventricle with obstructive hydrocephalus. Preoperative angiography revealed a feeding artery from the right medial posterior choroidal artery (MPChA). The feeding artery was effectively embolized, and the patient subsequently received open resection of the tumor. Pathology revealed features consistent with atypical choroid plexus papilloma (CPP).

LESSONS Choroid plexus tumors are often hypervascular, which can often make gross total resection (GTR) difficult. Preoperative angiography of tumors identifies feeding vessels, and embolization has the potential to decrease perioperative blood loss and allow for a higher GTR rate. Third ventricular CPPs appear to invariably receive vascular supply from the MPChA, arising from the right posterior cerebral artery. Embolization followed by resection of a choroid plexus tumor in the third ventricle is an effective treatment strategy.
\end{abstract}

https://thejns.org/doi/abs/10.3171/CASE21516

KEYWORDS choroid plexus papilloma; pediatrics; embolization; third ventricular tumor

Choroid plexus tumors are rare neoplasms, accounting for $<1 \%$ of all brain tumors. ${ }^{1}$ These tumors are pathologically divided into World Health Organization (WHO) grade I choroid plexus papilloma (CPP), WHO grade II atypical CPP (aCPP), and WHO grade III choroid plexus carcinomas (CPCs). ${ }^{2}$ These tumors are typically found in the lateral ventricle in children and more commonly in the fourth ventricle in adults. Choroid plexus tumors are much less frequently found in the third ventricle and have been reported in several case reports in the literature. ${ }^{3,4}$ Presurgical embolization of tumors in this region have been infrequently described. ${ }^{5-7}$ We present a case of a large third ventricular aCPP treated by presurgical embolization followed by open resection. We discuss the clinical course and review the current literature in this rare case.

\section{Illustrative Case}

A 6-month-old full-term girl presented with 1 week of irritability and hypotonia. Her mother noted that the patient was more irritable and had decreased movement of the lower extremities with an inability to roll side to side. There was no significant medical, social, or family history. The patient first presented to Children's Hospital Orange County with stable vital signs. The examination was notable for macrocephaly (head circumference $49.5 \mathrm{~cm}$, 100th percentile) and sundowning of the eyes.

Computed tomography of the head showed findings of significant hydrocephalus, prompting further evaluation with magnetic resonance imaging (MRI). MRI (Fig. 1) revealed a well-circumscribed mass in the third ventricle that extended through bilateral foramen of Monro into the lateral ventricles. The mass measured $4.8 \mathrm{~cm}$ anteroposterior by $3.9 \mathrm{~cm}$ transverse by $5.8 \mathrm{~cm}$ craniocaudal. The lesion demonstrated isointense T1 signal, heterogeneous T2 signal, intense enhancement, and diffusion restriction. There was associated ventriculomegaly involving the dilated third ventricle and lateral ventricles, with biventricular diameter of approximately $80 \mathrm{~mm}$.

ABBREVIATIONS aCPP = atypical choroid plexus papilloma; $\mathrm{CPC}=$ choroid plexus carcinoma; $\mathrm{CPP}=$ choroid plexus papilloma; EVD = external ventricular drain; GTR = gross total resection; MPChA = medial posterior choroidal artery; MRI = magnetic resonance imaging; WHO = World Health Organization. INCLUDE WHEN CITING Published January 31, 2022; DOI: 10.3171/CASE21516.

SUBMITTED September 10, 2021. ACCEPTED November 15, 2021.

(c) 2022 The authors, CC BY-NC-ND 4.0 (http://creativecommons.org/licenses/by-nc-nd/4.0/). 

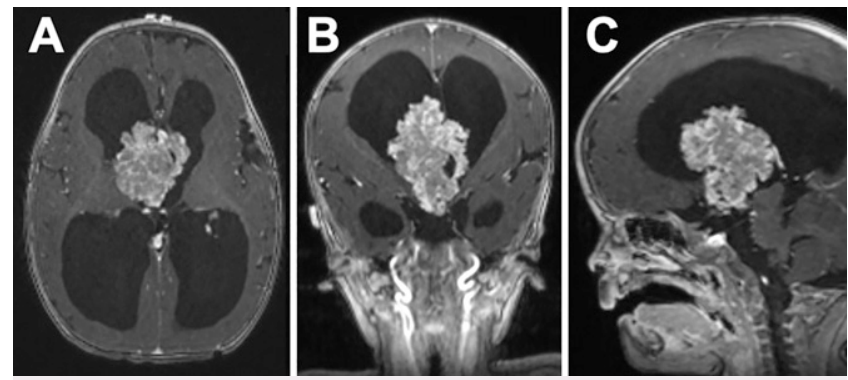

FIG. 1. Preoperative MRI. T1-weighted postcontrast sequences of axial (A), coronal (B), and sagittal (C) views demonstrating an enhancing tumor in the third ventricle.

\section{Treatment Course}

Because of symptomatic hydrocephalus, an external ventricular drain (EVD) was recommended. A biopsy was offered at the same time to establish diagnosis and determine the best treatment strategy. The patient received stereotactic biopsy and EVD placement, with pathology consistent with CPP. The decision was made to proceed with a diagnostic cerebral angiogram with possible embolization followed by resection.

\section{Embolization}

Angiography was performed 2 days later. The right femoral artery was accessed with a 4-Fr vascular sheath. A right medial posterior choroidal artery (MPChA) was noted to provide feeders to the tumor (Fig. 2). We proceeded with presurgical embolization. The 4-Fr angled nontapered diagnostic catheter was used as the guiding catheter. Under real-time roadmap images, a microcatheter system (Marathon microcatheter and Hybrid 008 microguidewire) was carefully navigated into the left vertebral artery, followed by the basilar artery, and finally the right P1 segment. Superselective angiography of the feeding artery was performed (Fig. 3). After obtaining stable microcatheter positioning, the microcatheter was primed with dimethyl sulfoxide. Then the feeding artery was embolized with Onyx 18 liquid embolic agent. The microcatheter was removed. Postembolization angiography of the left vertebral artery (cerebral view) was performed, showing satisfactory occlusion of the feeding artery.

\section{Surgery}

The patient received resection of tumor immediately after embolization. A right frontal entry was selected based on stereotactic guidance, and a transcortical, transsulcal entry was used to approach the tumor. A microscope was used at that point, and circumferential dissection of the tumor was completed with bipolar electrocautery and suction. The tumor rested in the third ventricle and was noted to travel into the left foramen of Monro and lateral ventricle. A section of the tumor was tethered to the MPChA, which was filled with embolic material. The vascular trunk was coagulated with bipolar electrocautery and cut, and the tumor was successfully removed. An EVD was left in the surgical cavity. The EVD was monitored for

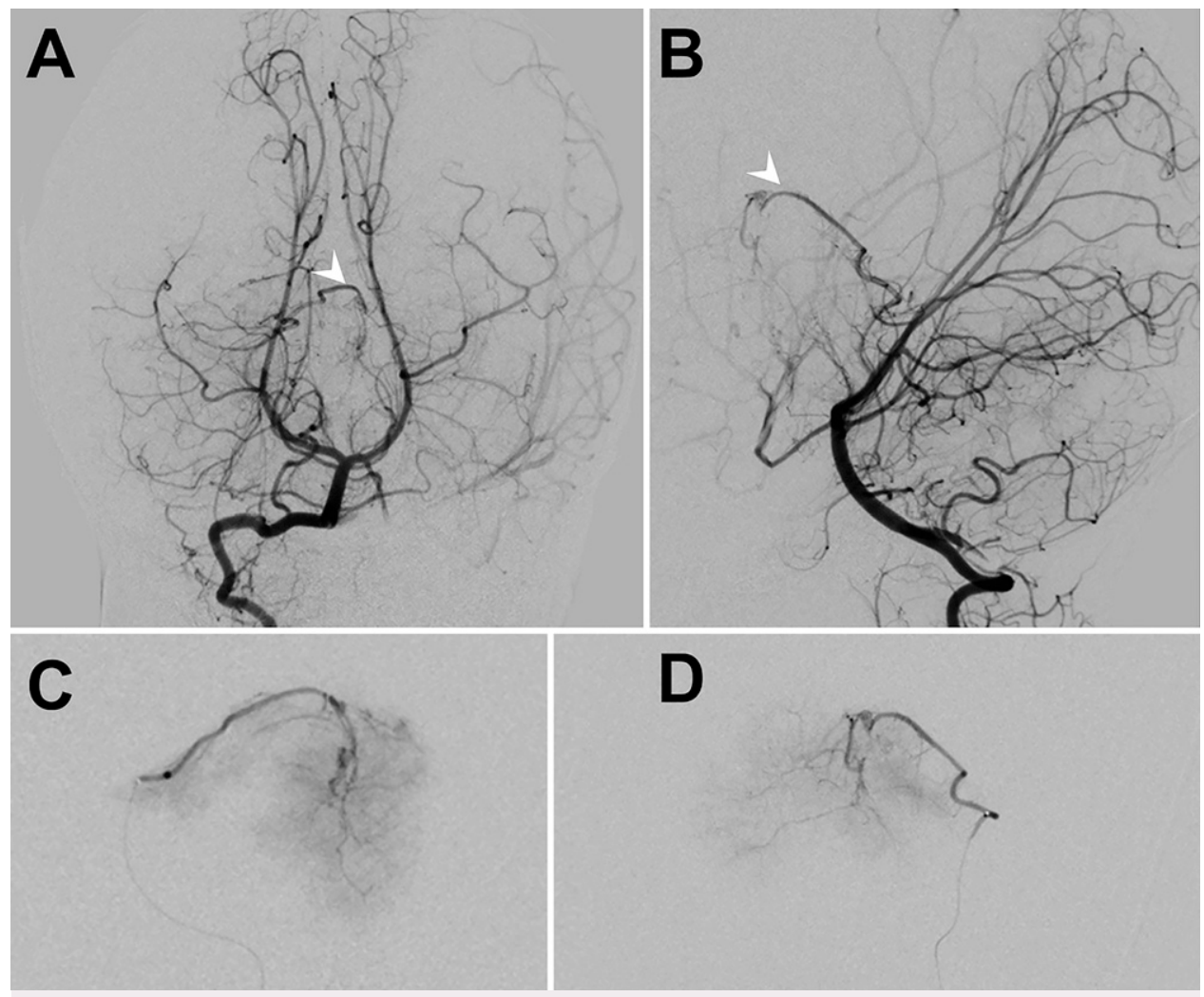

FIG. 2. Preoperative digital subtraction angiograms. Right vertebral artery injection with anteroposterior (A) and lateral (B) views revealing a feeding artery (white arrowheads) from the right P1 segment. Anteroposterior (C) and lateral (D) views of a superselective angiogram of the feeding artery. 

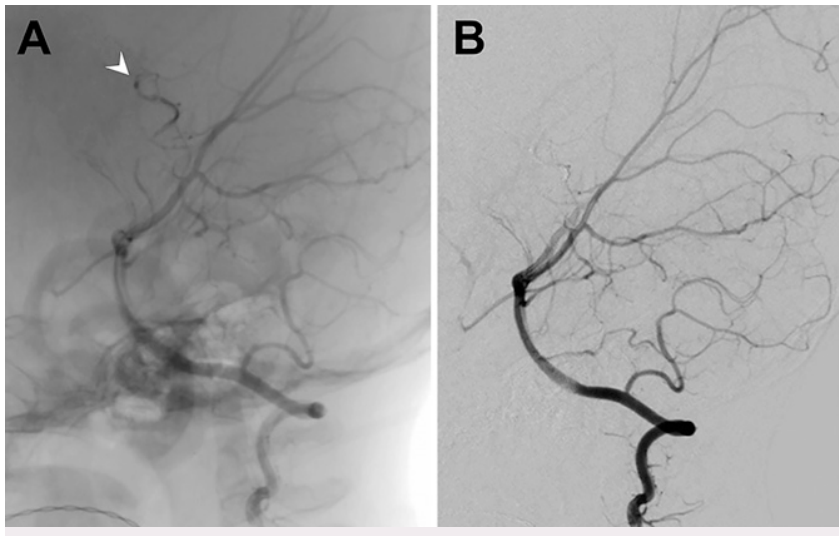

FIG. 3. Postembolization angiogram. A: Lateral angiogram of a right vertebral artery injection with embolization material within feeding artery (white arrowhead). B: Digital subtraction angiogram demonstrating occlusion of the previously noted feeding artery.

several days; because of continued elevated intracranial pressure, we proceeded with shunt placement on postoperative day 7 .

\section{Pathology}

Microscopic sections of the tumor show features consistent with aCPP (WHO grade II). The lesion was composed of well-developed papillary architecture without areas of solid growth or blurring of the papillae (Fig. 4). The papillae were mainly lined with a single layer of cuboidal to columnar cells with multifocal nuclear crowding and mild cytological atypia. Scattered mitotic figures (up to 4 mitoses per 10 high-power field) and focal necrosis were seen.

\section{Follow-Up}

Follow-up MRI (Fig. 5) was performed 9 months postoperatively, with no evidence of residual neoplasm. The patient was seen in clinic the same day and was noted to be doing well. She had been meeting all developmental milestones. She will continue receiving surveillance MRI.

\section{Discussion}

Tumors of the choroid plexus are rare and typically arise from the lateral ventricles in children and fourth ventricles in adults. ${ }^{8}$ These tumors are derived from choroid plexus epithelium and present as CPP, aCPP, and CPC. ${ }^{2}$ Choroid plexus tumors have been

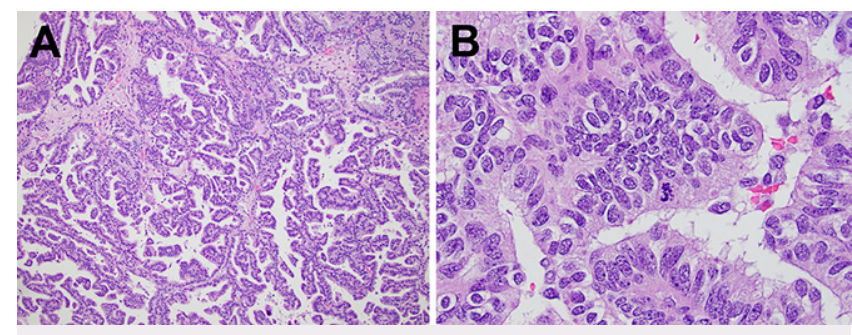

FIG. 4. A and B: Hematoxylin and eosin staining demonstrates histological features of the tumor composed of papillary structures. B:

Nuclear crowding with increased mitotic activity. Original magnification $\times 100($ A $)$ and $\times 200(B)$.
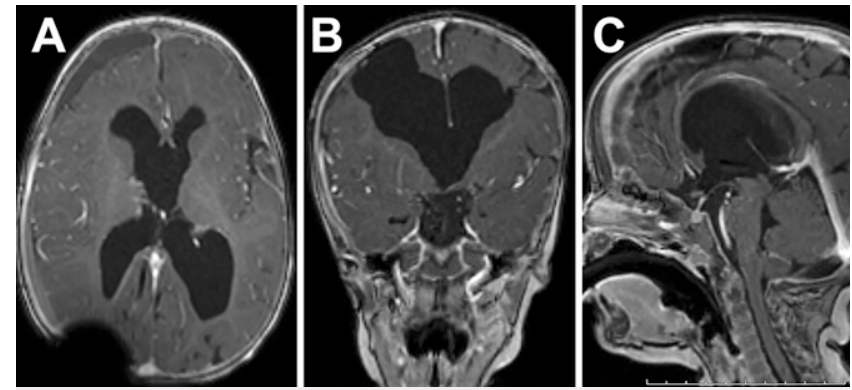

FIG. 5. Nine-month postoperative MRI. T1-weighted postcontrast sequences of axial (A), coronal (B), and sagittal (C) views. No enhancement noted, suggesting no residual tumor.

shown to cause hydrocephalus by both obstructing normal cerebrospinal fluid pathways and overproducing cerebrospinal fluid. ${ }^{9}$ Gross total resection (GTR) is the treatment of choice and usually has good outcome for choroid plexus tumors, although CPCs are more aggressive and have a higher rate of recurrence. ${ }^{8}$ Surgical approaches to third ventricular tumors include transcallosal, transfrontal, and transforaminal routes and supracerebellar infratentorial routes. ${ }^{4,10}$ The surgical approach should be tailored to individual cases and depends on location, size, vascular supply, and extension of tumor.

\section{Observations}

Choroid plexus tumors are often hypervascular, which may make GTR more difficult. Preoperative embolization of tumors has the potential to decrease perioperative blood loss and allow for a higher chance of GTR. ${ }^{6,7}$ It has the potential to shorten operative time and decrease the length of anesthesia, which are important considerations in the pediatric population. Cannulation of a feeding artery and embolization may be technically difficult because of the increased tortuosity and small caliber of arteries, such as the posterior choroidal artery. Advances in endovascular materials and techniques have allowed for safer procedures and better access to smaller and more distal vasculature. Special consideration should be taken for endovascular techniques in pediatric patients, who are smaller and have more delicate tissue. A smaller sheath (4 Fr) and gentler techniques should be used. The sensitivity of children to even modest doses of radiation should also be considered, and exposure to radiation should be minimized.

Few studies in the literature have reported successful embolization of choroid plexus tumors. ${ }^{6,7}$ Even fewer authors report successful embolization of third ventricular choroid plexus tumors. ${ }^{5,6}$ Wind et al. reported a third ventricular CPP with vascular supply also from a MPChA arising from the right posterior cerebral artery. The patient's tumor was embolized at 3 months of age with intention to surgically resect at a later age. Serial MRI showed steady regression of the tumor, and there was no visible lesion 7 months after embolization. Do et al. report a third ventricular CPP fed by both left lateral posterior and right medial posterior choroidal arteries. Both supplying arteries were embolized when the patient was 8 months old and was followed by craniotomy for transcallosal tumor resection. Tumors of the third ventricle appear to invariably receive supply from the MPChA. ${ }^{6}$

\section{Lessons}

Third ventricular choroid plexus tumors are infrequently reported and can cause symptoms from hydrocephalus. GTR is the treatment 
of choice. Preoperative angiography can provide information on vascular supply and an opportunity to embolize feeding arteries. Third ventricular CPPs are supplied by the MPChA and can be effectively embolized. Effective embolization can facilitate resection by decreasing blood loss and increasing the chance of GTR.

\section{References}

1. Ostrom QT, Patil N, Cioffi G, Waite K, Kruchko C, Barnholtz-Sloan JS. CBTRUS statistical report: primary brain and other central nervous system tumors diagnosed in the United States in 20132017. Neuro Oncol. 2020;22(12 suppl 2):iv1-iv96.

2. Misiolek KA, Osborn ZG, Hauser N, Thomas D, Goodman JF, Fulkerson DH. Rapidly growing, multifocal, benign choroid plexus tumor in an infant: case report. $J$ Neurosurg Pediatr. Published online February 22, 2019. doi: 10.3171/2018.12.PEDS18453.

3. Pfeifer CM, Wong K, Malireddy R, Van Tassel D, Veltkamp DL, Cornejo P. Third ventricle choroid plexus papilloma: 2 cases. Radiol Case Rep. 2020;15(5):454-456.

4. Gupta P, Sodhi KS, Mohindra S, Saxena AK, Das A, Khandelwal N. Choroid plexus papilloma of the third ventricle: a rare infantile brain tumor. J Pediatr Neurosci. 2013;8(3):247-249.

5. Wind JJ, Bell RS, Bank WO, Myseros JS. Treatment of third ventricular choroid plexus papilloma in an infant with embolization alone. J Neurosurg Pediatr. 2010;6(6):579-582.

6. Do HM, Marx WF, Khanam H, Jensen ME. Choroid plexus papilloma of the third ventricle: angiography, preoperative embolization, and histology. Neuroradiology. 2001;43(6):503-506.

7. Haliasos N, Brew S, Robertson F, Hayward R, Thompson D, Chakraborty A. Preoperative embolisation of choroid plexus tumours in children: part I. Does the reduction of perioperative blood loss affect the safety of subsequent surgery? Childs Nerv Syst. 2013;29(1):65-70

8. Rickert $\mathrm{CH}$, Paulus W. Tumors of the choroid plexus. Microsc Res Tech. 2001;52(1):104-111.

9. Eisenberg HM, McComb JG, Lorenzo AV. Cerebrospinal fluid overproduction and hydrocephalus associated with choroid plexus papilloma. J Neurosurg. 1974;40(3):381-385.

10. Apuzzo ML, Chikovani OK, Gott PS, et al. Transcallosal, interfornicial approaches for lesions affecting the third ventricle: surgical considerations and consequences. Neurosurgery. 1982;10(5): $547-554$

\section{Disclosures}

The authors report no conflict of interest concerning the materials or methods used in this study or the findings specified in this paper.

\section{Author Contributions}

Conception and design: Xu, Chrislip, Nael, Loudon. Acquisition of data: $\mathrm{Xu}$, Suzuki, Nael, Loudon. Analysis and interpretation of data: Xu, Nael, Loudon. Drafting the article: $\mathrm{Xu}$, Loudon. Critically revising the article: all authors. Reviewed submitted version of manuscript: Xu, Nael, Loudon. Approved the final version of the manuscript on behalf of all authors: Xu. Administrative/technical/material support: Xu, Suzuki, Nael. Study supervision: Xu, Suzuki, Nael, Loudon.

\section{Correspondence}

Jordan C. Xu: University of California, Irvine Medical Center, Orange, CA. jcxu@hs.uci.edu. 